\title{
A D-band SPST switch using parallel-stripline swap with defected ground structure
}

\author{
Jiang Luo ${ }^{1}$, Jin He$^{1 \mathrm{a})}$, Alit Apriyana ${ }^{2}$, Guangyin Feng ${ }^{2}$, \\ and Qijun Huang ${ }^{1 b)}$ \\ ${ }^{1}$ School of Physics and Technology, Wuhan University, \\ 299 Bayi Road, Wuhan, 430072, China \\ ${ }^{2}$ School of Electrical and Electronic Engineering, Nanyang Technological \\ University, 50 Nanyang Avenue, 639798, Singapore \\ a) jin.he@whu.edu.cn \\ b)huangqj@whu.edu.cn
}

\begin{abstract}
In this paper, we propose a D-band switch design using a parallel-stripline swap hybrid coupler with defected ground structure (DGS). Compared to the conventional transmission line (TL) with high insertion loss at high frequency, the equivalent capacitance introduced by the coupler gap leads to series resonance condition which results in lower insertion loss at the higher frequency. The hybrid coupler enables two additional degrees of design freedom since the capacitance and inductance can be tuned by the structural dimension of the coupler. The fabricated switch with core area of $0.0036 \mathrm{~mm}^{2}$ shows measured insertion loss of 1.6-3 dB from $110-134 \mathrm{GHz}$, return loss of higher than $10 \mathrm{~dB}$ and isolation level around $16 \mathrm{~dB}$.
\end{abstract}

Keywords: CMOS, parallel-stripline swap hybrid coupler, defected ground structure (DGS), single-pole-single-throw (SPST) switch, D-band

Classification: Integrated circuits

\section{References}

[1] A. Poh and Y. P. Zhang: "Design and analysis of transmit/receive switch in triple-well CMOS for MIMO wireless systems," IEEE Trans. Microw. Theory Techn. 55 (2007) 458 (DOI: 10.1109/TMTT.2006.890510).

[2] M. Uzunkol and G. M. Rebeiz: "140-220 GHz SPST and SPDT switches in $45 \mathrm{~nm}$ CMOS SOI," IEEE Microw. Wireless Compon. Lett. 22 (2012) 412 (DOI: 10.1109/LMWC.2012.2206017).

[3] Z. M. Tsai, et al.: "FET-integrated CPW and the application in filter synthesis design method on traveling-wave switch above $100 \mathrm{GHz}$," IEEE Trans. Microw. Theory Techn. 54 (2006) 2090 (DOI: 10.1109/TMTT.2006.873629).

[4] B. Zhang, et al.: "A switch-based ASK modulator for $10 \mathrm{Gbps} 135 \mathrm{GHz}$ communication by $0.13-\mu \mathrm{m}$ MOSFET," IEEE Microw. Wireless Compon. Lett. 22 (2012) 415 (DOI: 10.1109/LMWC.2012.2205227).

[5] U. Yodprasit, et al:: "D-band 3.6-dB-insertion-loss ASK modulator with 19.5$\mathrm{dB}$ isolation in 65-nm CMOS technology," Asia-Pacific Microwave Conference (APMC) (2010) 1853. 
[6] X. D. Deng, et al.: "A W-band single-pole single-throw switch using on-chip rectangular coaxial transmission line in $0.13-\mu \mathrm{m}$ CMOS technology," AsiaPacific Microwave Conference (APMC) 2 (2015) 1 (DOI: 10.1109/APMC. 2015.7413200).

[7] J. He, et al.: "Analysis and design of 60-GHz SPDT switch in 130-nm CMOS," IEEE Trans. Microw. Theory Techn. 60 (2012) 3113 (DOI: 10.1109/TMTT. 2012.2211380).

[8] A. A. A. Agung, et al.: "An ultrawideband SPST switch using defected ground structure low pass filter in 65-nm CMOS technology," Int. J. RF Microw. Comput.-Aided Eng. 25 (2015) 758 (DOI: 10.1002/mmce.20912).

[9] L. Chiu and Q. Xue: "A parallel-strip ring power divider with high isolation and arbitrary power-dividing ratio," IEEE Trans. Microw. Theory Techn. 55 (2007) 2419 (DOI: 10.1109/TMTT.2007.908669).

[10] M. B. Shifrin, et al:: "Monolithic FET structure for high-power control component applications," IEEE Trans. Microw. Theory Techn. 37 (1989) 2134 (DOI: 10.1109/22.44132).

[11] C. Tinella, et al.: "A high-performance CMOS-SOI antenna switch for the 2.5-5-GHz band," IEEE J. Solid-State Circuits 38 (2003) 1279 (DOI: 10.1109/ JSSC.2003.813289).

[12] V. Issakov, et al:: "Extension of thru de-embedding technique for asymmetrical and differential devices," IET Circuits Dev. Syst. 3 (2009) 91 (DOI: 10.1049/ iet-cds.2008.0276).

\section{Introduction}

Single-pole-single-throw (SPST) switch is a crucial building block of single-pole multiple-throw (SPMT) switches for time-domain-duplexing (TDD)-based frontends, switched-beam arrays and multiple-input and multiple-output (MIMO) systems $[1,2,3]$. The main challenge in CMOS millimeter-wave (mm-wave) switches design lies in compensation of the parasitic capacitances of the control transistors. This parasitic greatly affects the switch performances, especially the insertion loss (IL), return loss (RL) and isolation (ISO). Based on the inductive elements that are used to compensate the parasitic capacitances, there are some compensation methods demonstrated for mm-wave switches. The most common method is to employ $\lambda / 4$ transmission lines $[3,4,5]$. The use of TLs as the compensating element has the benefit of wide-band characteristic due to its inherent broadband properties. However, the compensation method with TLs occupies large silicon area, leading to higher fabrication cost. To achieve more compact silicon area, $\mathrm{He}$ et al. proposed using a series inductor to replace TLs [7], the method with inductor can save some silicon area but it is hard to provide a wide-band operating frequency range because of high $Q$ inductors. In addition, the inductor causes excessive loss when the operating frequency is higher than $100 \mathrm{GHz}$ due to skin effect [7]. To alleviate these issues, a low-pass filter with defected ground structure is adopted in the SPST switch design in [8]. The technique has some advantages that compensating the parasitic capacitances more effectively and consuming less silicon area; however, the low-pass filter topology has the problem of operating frequency limitation at high frequency because of its natural low-pass characteristics. Thus, a new architecture for mm-wave switches is desired. 
In this paper, a parallel-stripline swap hybrid coupler [9] with defected ground structure (DGS) is proposed as an alternative compensation method. The proposed method is demonstrated in a D-band SPST switch design using $65-\mathrm{nm}$ bulk CMOS process. This fabricated switch achieves a minimum IL of $1.6 \mathrm{~dB}$ with a core size of only $0.0036 \mathrm{~mm}^{2}$. The paper is organized as follows. Section II describes the analysis and design of the proposed SPST switch. The measurement results that verify the design method are discussed in section III. Finally, the paper is concluded in Section IV.

\section{Circuit analysis and design}

\subsection{Parallel-stripline swap hybrid coupler with defected ground structure}

Fig. 1 shows the topology of parallel-stripline swap hybrid coupler with defected ground structure, which consists of two transmission lines at both sides and a defected ground plane. A swap core is constructed using two vertical metallic vias, which are employed to bridge the upper metal layer and bottom metal layer. The defected ground plane consists of two large rectangular slots that are connected by a small gap. For a conventional TL, the current return path on the ground plane is the anti-phase of the current path on microstrip line. The surface current distributions of the hybrid coupler are plotted in Fig. 1. The opening in the ground plane disturbs the return current distribution and thus, the current is confined to the edges of two polygon slots. This results in the enhancement of the effective inductance due to the increase in the length of the current return path.

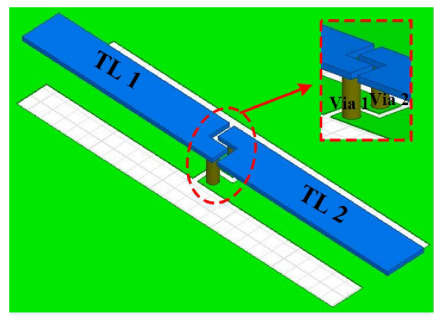

(a)

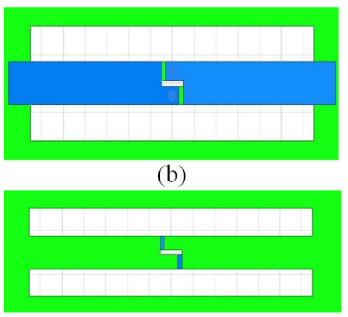

(c)

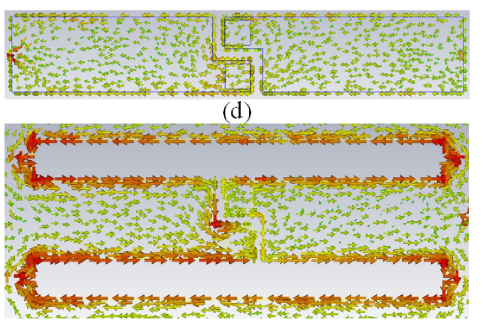

(e)

Fig. 1. Illustration of parallel-stripline swap hybrid coupler with a defected ground structure: (a) 3-D view; (b) Top view; (c) Bottom view; (d) Surface current distribution on the upper metal layer; (e) Surface current distribution on defected ground plane.

At the desired frequency range, the input signal can be coupled to the output port through the coupling capacitance that is introduced by the gap between TL-1 and TL-2 of the hybrid coupler. Hence, this coupling capacitance determines the resonance frequency of the hybrid coupler. Since the capacitance can be tuned by changing the gap dimension, we have an additional degree of freedom to control the frequency response of the coupler and further the switch performance. Unlike the conventional transmission line, the coupling capacitance of the hybrid coupler introduces series resonance condition (zeros) at high frequency, hence we can achieve higher operating frequency with lower loss. Fig. 2 shows the comparison of simulated IL between the hybrid coupler with DGS and conventional TL. As 
we can see, hybrid coupler with DGS shows more superior insertion loss performance at high frequency than conventional TL. Hence, we use this structure as alternative compensation method to replace the conventional transmission line in SPST switch design.

In addition, defected ground structure enhances the equivalent inductance of the hybrid coupler, which can be used to compensate the parasitic capacitance of control transistors of the switch. Since the inductance can be tuned by changing the size of the defected area, we have the additional parameter to tune the overall response of the switch. Due to its planar structure, simple fabrication process, and compatibility with CMOS technology, it provides more compact inductive components with low cost for fully integrated advanced D-band systems.

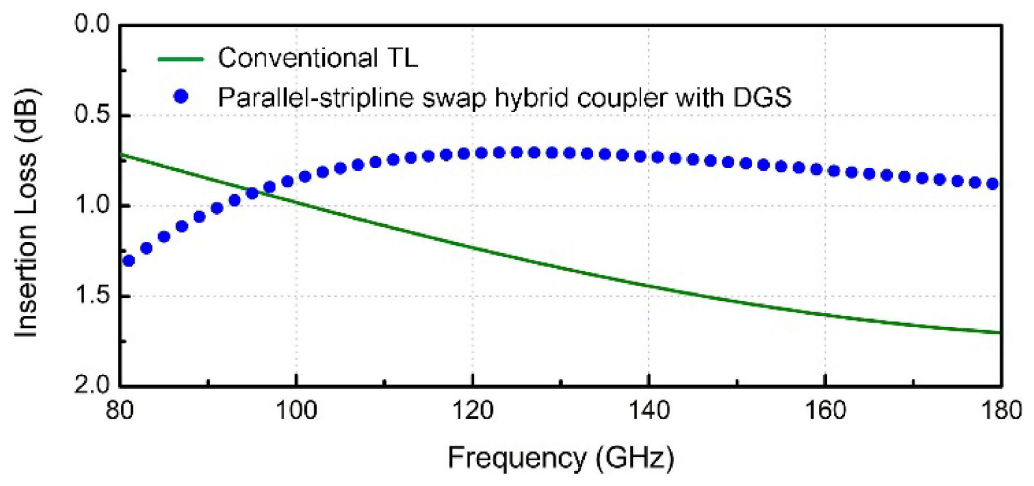

Fig. 2. The EM simulated IL of parallel-stripline swap hybrid coupler with DGS and conventional TL at the same physical size.

\subsection{Circuit implementation}

Table I. Target specifications for the SPST switch

\begin{tabular}{|c|c|}
\hline Design Parameter & Value \\
\hline Operating frequency $(\mathrm{GHz})$ & $110-130$ \\
\hline Insertion Loss $(\mathrm{dB})$ & $<3$ \\
\hline Return Loss $(\mathrm{dB})$ & $>10$ \\
\hline Isolation $(\mathrm{dB})$ & $>15$ \\
\hline Input $P_{1 \mathrm{~dB}}(\mathrm{dBm})$ & $>10$ \\
\hline Core Area $\left(\mathrm{mm}^{2}\right)$ & $<0.01$ \\
\hline$V_{\text {ctrl }}(\mathrm{V})$ & $0 / 1.2$ \\
\hline
\end{tabular}

A switch is required with the performance of low insertion loss, high isolation, large power-handling capability, and operating bandwidth. Containing great spectrum resources from $110 \mathrm{GHz}$ to $170 \mathrm{GHz}$, D-band is ideally suitable for shortrange and extremely high speed wireless systems. The target of designing the SPST switch is to construct the single-pole-double-throw (SPDT) transmit/receive (T/R) switch for $120 \mathrm{GHz}$ wireless system in future work, which can be employed for the purpose of sharing a single antenna between the transmitter and receiver, thus leading to a more compact solution for fully integrated D-band systems in CMOS. Our target specifications for the proposed SPST switch at $120 \mathrm{GHz}$ are an insertion 
loss of $<3 \mathrm{~dB}$, an isolation of $>15 \mathrm{~dB}$, and input $P_{1 \mathrm{~dB}}$ of $>10 \mathrm{dBm}$. The detailed target specifications for the 120-GHz SPST switch are given in Table I.

The proposed switch design is shown in Fig. 3, which is composed of the parallel-stripline swap hybrid coupler with DGS, two shunt nMOS transistors $T_{1}$ and $T_{2}$, and two biasing resistors $R_{\mathrm{G} 1}$ and $R_{\mathrm{G} 2}$ at transistor gate terminals. The enhanced coupler's inductance is used to compensate the shunt parasitic capacitances that are introduced by the control transistors $T_{1}$ and $T_{2}$. The transistors $T_{1}$ and $T_{2}$ are used to turn on or turn off the switch, depending on the state of the control voltage $\left(V_{\text {ctrl }}\right)$. When $V_{\text {ctrl }}$ is set to $0 \mathrm{~V}$, both transistors $T_{1}$ and $T_{2}$ are offstate. Then, the signal at Port 1 can be passed to Port 2 through the coupler. When the $V_{\text {ctrl }}$ is $1.2 \mathrm{~V}$, both transistors $T_{1}$ and $T_{2}$ are on-state and thereby both ports are shorted to the ground. Ideally, there is no signal passing through from Port 1 to Port 2. The finger width of $2.5-\mu \mathrm{m}$ and a minimum gate length of $60-\mathrm{nm}$ are chosen for both transistors $T_{1}$ and $T_{2}$ to obtain the optimum compromise between isolation and insertion loss performance. The gate resistors $R_{\mathrm{G} 1}$ and $R_{\mathrm{G} 2}$ are used to bootstrap the gate at RF. By bootstrapping the gate, it will be floating at RF and hence its gate-to-source and gate-to-drain potential will be relatively constant.

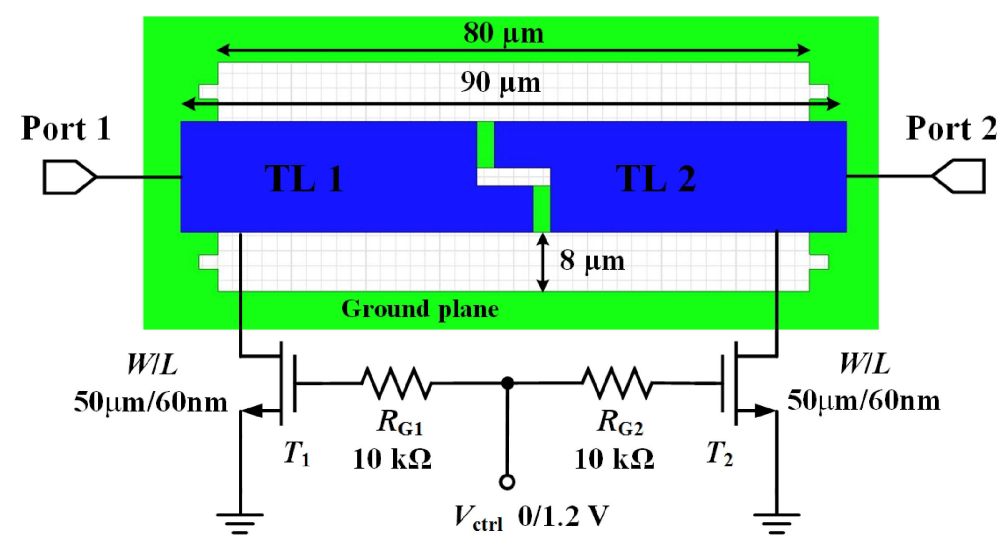

Fig. 3. Schematic of proposed SPST switch using parallel-stripline swap hybrid coupler with DGS.

The power-handling capability or linearity of the SPST switch is evaluated by the input $1-\mathrm{dB}$ compression point $\left(P_{1 \mathrm{~dB}}\right)$, which is directly associated with the offstate shunt transistors. With large resistors $R_{\mathrm{G} 1}$ and $R_{\mathrm{G} 2}$ floating the gates of transistor $T_{1}$ and $T_{2}$ at $\mathrm{RF}$, the voltage amplitude of the signal can be effectively increased to $2 V_{t h}$, where $V_{t h}$ is the threshold voltage of the nMOS transistor $[10,11]$. Hence, the input $P_{1 \mathrm{~dB}}$ of switch with gate biasing resistor is derived in $\mathrm{dBm}[11]$ as:

$$
P_{1 d B}=10 \log \left(\frac{2 V_{t h}^{2}}{\left|Z_{\text {in }}\right|}\right)+30
$$

Where $Z_{\text {in }}$ is input impedance. For the 65 -nm bulk CMOS process, the typical value of $V_{t h}$ is around $0.5-0.6 \mathrm{~V}$, thereby the calculated input $P_{1 \mathrm{~dB}}$ is around $10-11.6 \mathrm{dBm}$ for $Z_{\text {in }}=50 \Omega$. As illustrated in Fig. 4, input $P_{1 \mathrm{~dB}}$ is enhanced with the increasing of $R_{G}$ until $R_{G}$ is higher than $100 \Omega$ where $P_{1 \mathrm{~dB}}$ is almost constant due to the turnon of the junction diodes. 
Larger gate biasing resistor provides better DC isolation on the gate and improves switch linearity, but at the expense of longer switching times because the gate capacitance must be charged and discharged through high resistance values of gate biasing resistor. A typical range of values for gate biasing resistor is $5 \mathrm{k} \Omega$ to $15 \mathrm{k} \Omega$. Fig. 5 shows the detailed rise time $t_{\mathrm{r}}$ (from $10 \%$ up to $90 \%$ of the maximum output swing) and fall time $t_{\mathrm{f}}$ (from $90 \%$ down to $10 \%$ of the maximum output swing) of the output signal when both gate biasing resistors $R_{\mathrm{G} 1}$ and $R_{\mathrm{G} 2}$ increase from $6 \mathrm{k} \Omega$ to $14 \mathrm{k} \Omega$. The switch output voltage takes longer time to reach its maximum value for larger $R_{\mathrm{G}}$. The switching speed could be improved by decreasing gate bias resistors $R_{\mathrm{G} 1}$ and $R_{\mathrm{G} 2}$. To tradeoff between input $P_{1 \mathrm{~dB}}$ and switching response speed performance, the resistance value of $R_{\mathrm{G} 1}$ and $R_{\mathrm{G} 2}$ is chosen to $10 \mathrm{k} \Omega$.

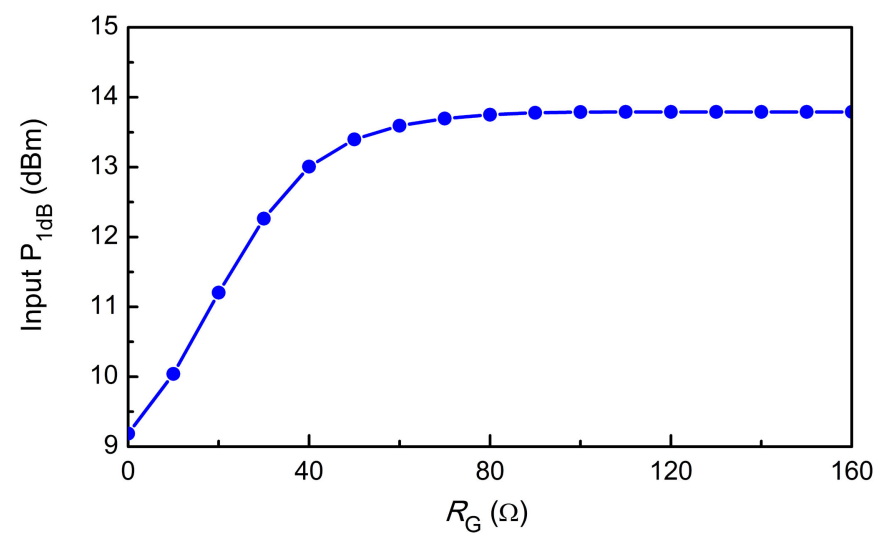

Fig. 4. Input referred $P_{1 \mathrm{~dB}}$ compression point as function of the changes in the values of the gate resistor $\left(R_{G}\right)$.

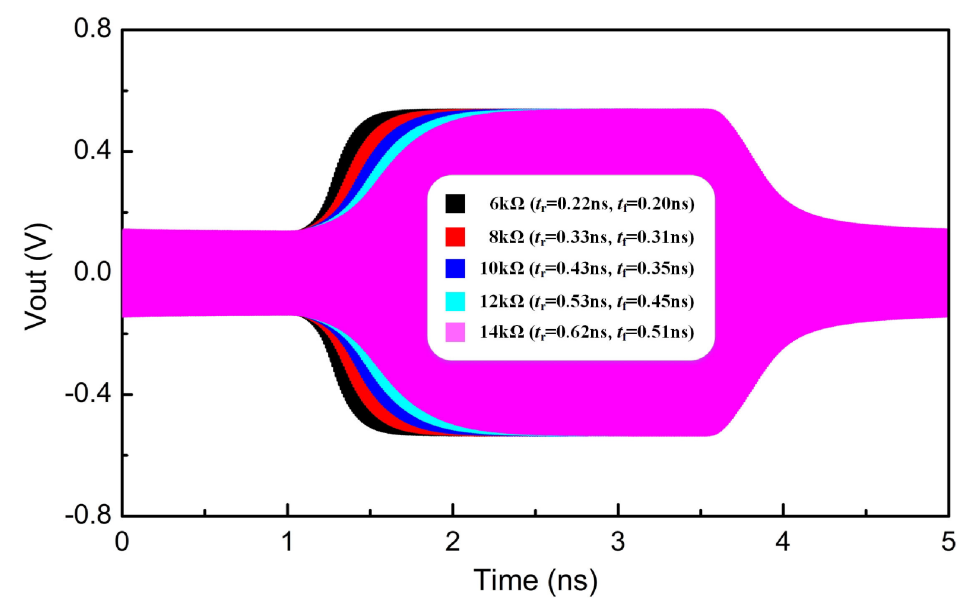

Fig. 5. Switching speed performance as function of the changes in the values of the gate resistor $\left(R_{G}\right)$.

The cross-section schematic of the 65-nm bulk CMOS process is illustrated in Fig. 6(a). From bottom to top, this process includes one polymer layer, seven thin metal layers $\left(\mathrm{M}_{1}\right.$ through $\left.\mathrm{M}_{7}\right)$ and two thick metal layers $\left(\mathrm{M}_{8}\right.$ and $\left.\mathrm{M}_{9}\right)$. Note that the fat-wire of $M_{8}$ can achieve lower loss than $M_{9}$ although $M_{9}$ is higher than $M_{8}$ from the substrate, because $\mathrm{M}_{9}$ is aluminum while the other metal layers are copper. This technology provides two opinions of nMOS transistors, thin gate for the $1.2 \mathrm{~V}$ 
supply voltage and thick gate for a $2.5 \mathrm{~V}$ supply voltage, respectively. The nMOS transistor for low-power applications achieves a cutoff frequency $f_{\mathrm{T}}$ around $200 \mathrm{GHz}$ and a maximum oscillation frequency $f_{\text {MAX }}$ around $220 \mathrm{GHz}$, respectively. The thickness, dielectric constant and resistivity of the substrate are $737 \mu \mathrm{m}$, 11.9 and $1.5 \Omega \cdot \mathrm{cm}$, respectively.

In order to achieve more accurate performance of the switch, a 3-D physical model of the SPST switch that included the whole passive components is built in a 3-D full wave electromagnetic (EM) high-frequency structure simulator (HFSS), as plotted in Fig. 6(b). The coupler is implemented by using sub-top layer $\left(\mathrm{M}_{8}\right)$ as the transmission line and bottom layer $\left(\mathrm{M}_{1}\right)$ as the ground plane, as well as two square vertical metal vias. The $T$ type junction of $T_{1}$ and $T_{2}$ are used to connect to the drain of two shunt nMOS transistors $T_{1}$ and $T_{2}$. In this work, the design parameters including all passive components and interconnections are optimized using 3-D EM HFSS simulator and circuit co-simulations, which are depicted in Fig. $3 \& 6$.

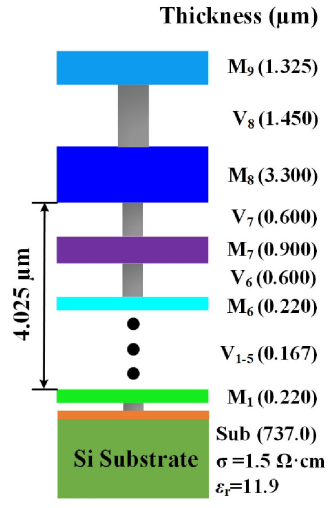

(a)

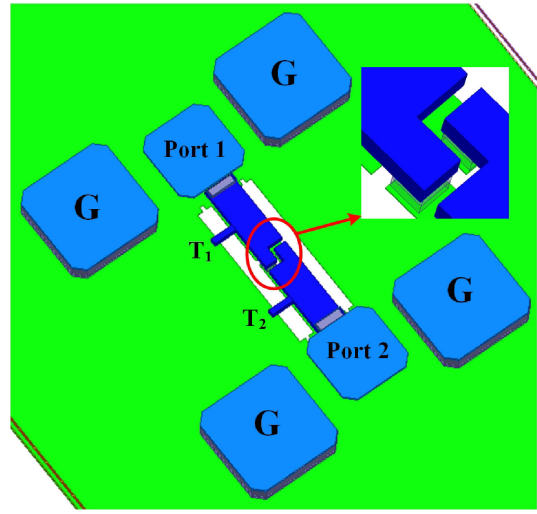

(b)

Fig. 6. (a) Cross-section schematic of the 65-nm bulk CMOS process; (b) The 3-D model of the SPST switch.

\section{Measurement and discussion}

The proposed SPST switch was fabricated using $65 \mathrm{~nm}$ bulk CMOS technology. The die microphotograph of the fabricated SPST switch is shown in Fig. 7. The whole chip occupies a silicon area of $0.0418 \mathrm{~mm}^{2}$ including all testing pads with a core size of only $0.0036 \mathrm{~mm}^{2}$. Note that the die thickness is about $0.767 \mathrm{~mm}$.

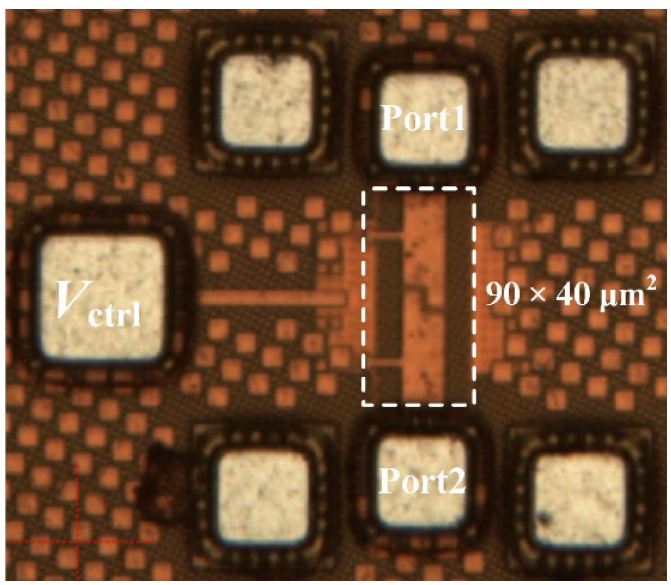

Fig. 7. Die micrograph of the proposed SPST switch. 
On-wafer measurements of two-port $S$-parameters were performed from 110 to $170 \mathrm{GHz}$ using Cascade Microtech Elite 300 probe station with Cascade $50-\mu \mathrm{m}$ pitch ground-signal-ground (G-S-G) waveguide probes, Agilent N5247A PNA-X Microwave Network Analyzer and two 110-170 GHz VDI's frequency extension modules. The system is calibrated using a short-open-load-thru (SOLT) probe-tip calibration technique on a Cascade Impedance Standard Substrate. The measured results are de-embedded using the thru de-embedding technique [12] to mitigate the effects of the interconnections and testing pads.
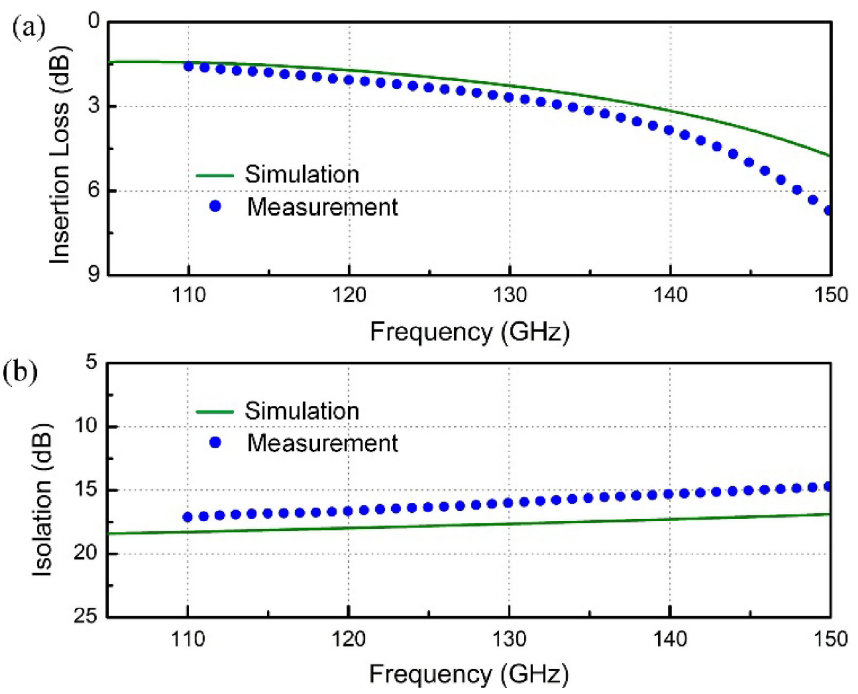

Fig. 8. Simulated and measured ILs and ISOs for the SPST switch.

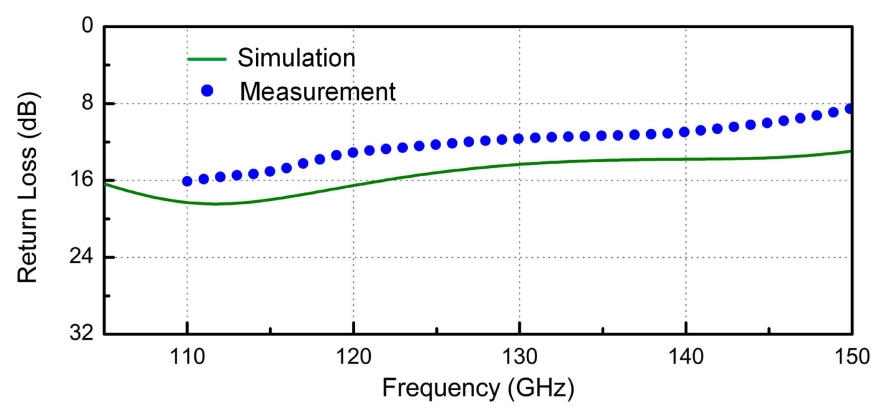

Fig. 9. Simulated and measured RLs for the SPST switch.

The simulated and measured ILs curve are plotted in Fig. 8(a). It can be seen that the switch has a minimum measured IL of $1.6 \mathrm{~dB}(1.4 \mathrm{~dB}$ simulated) at $110 \mathrm{GHz}$. The measured IL is $1.9 \mathrm{~dB}(1.72 \mathrm{~dB}$ simulated $)$ at $120 \mathrm{GHz}$. The switch exhibits a broadband characteristic and the measured IL is less than $3 \mathrm{~dB}$ from 110 to $134 \mathrm{GHz}$ (110 to $139 \mathrm{GHz}$ simulated). The simulated and measured ISOs between Port 1 and Port 2 are shown in Fig. 8(b). The measured switch achieves a maximum ISO of $17 \mathrm{~dB}(18.3 \mathrm{~dB}$ simulated $)$ at $110 \mathrm{GHz}$. The measured ISO is better than $15.0 \mathrm{~dB}(17 \mathrm{~dB}$ simulated $)$ from $110 \mathrm{GHz}$ to $150 \mathrm{GHz}$. The simulated and measured RLs are presented in Fig. 9. The measured RL is better than $10 \mathrm{~dB}$ ( $14 \mathrm{~dB}$ simulated) from $110 \mathrm{GHz}$ to $140 \mathrm{GHz}$. The measured $\mathrm{RL}$ is approximately 2 to $4 \mathrm{~dB}$ worse than the simulations at the frequency range of 110 to $140 \mathrm{GHz}$. 
The discrepancies between measured results and simulated results may be mainly due to the existence of the randomized dummy metallization which had not been fully considered in the initial simulation because of limited computing resource. These dummies metallization introduced additional parasitic capacitance, which affected the flow of ground current along DGS perimeter, as well as the inductance and $Q$ factor of the coupler. As the consequences, the RL and ISO are worse than the simulation results. When input and output matching is degraded, the measured IL also becomes worse.

The power-handling capability $P_{1 \mathrm{~dB}}$ is not measured at $120 \mathrm{GHz}$ due to power limitation of our available testing facilities. Because the maximum output power from frequency extension module is less than $-10 \mathrm{dBm}$ at D-band that was well below the input $P_{1 \mathrm{~dB}}$ of tested switch. The input $P_{1 \mathrm{~dB}}$ was simulated at $120 \mathrm{GHz}$ for the SPST switch. The simulated input $P_{1 \mathrm{~dB}}$ is around $12.8 \mathrm{dBm}$ at $120 \mathrm{GHz}$, as shown in Fig. 10. The calculated input $P_{1 \mathrm{~dB}}$ is around $11.3 \mathrm{dBm}$ when the value of $V_{\text {th }}$ and $V_{\text {ctrl }}$ are $580 \mathrm{mV}$ and $0 \mathrm{~V}$, respectively. The difference between simulated and calculated input $P_{1 \mathrm{~dB}}$ is mainly due to the input impedance is less than $50 \Omega$ when the input matching network is degraded because of the existence of randomized dummy fills.

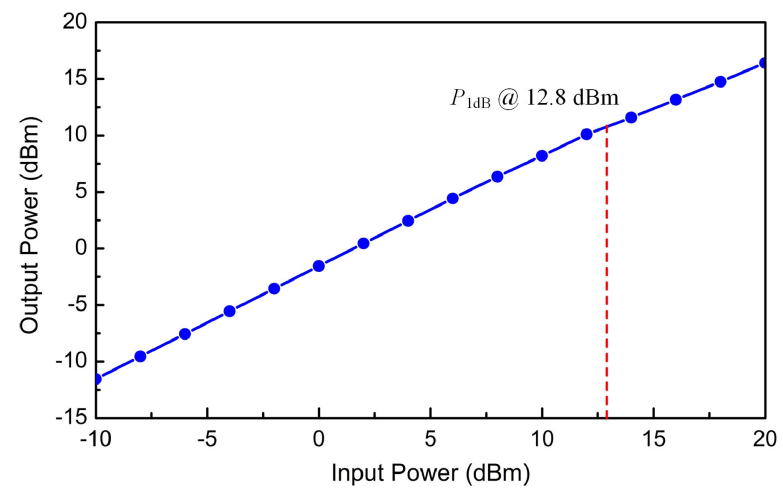

Fig. 10. Simulated input $P_{1 \mathrm{~dB}}$ for the SPST switch.

The switching speed of the SPST switch is simulated by employing an input sinusoid signal at $120 \mathrm{GHz}$, as well as a 500-MHz pulse control signal of $50 \%$ dutycycle. The rise time $t_{\mathrm{r}}$ and fall time $t_{\mathrm{f}}$ of the output signal are $0.43 \mathrm{~ns}$ and $0.35 \mathrm{~ns}$, respectively, as shown in Fig. 11.

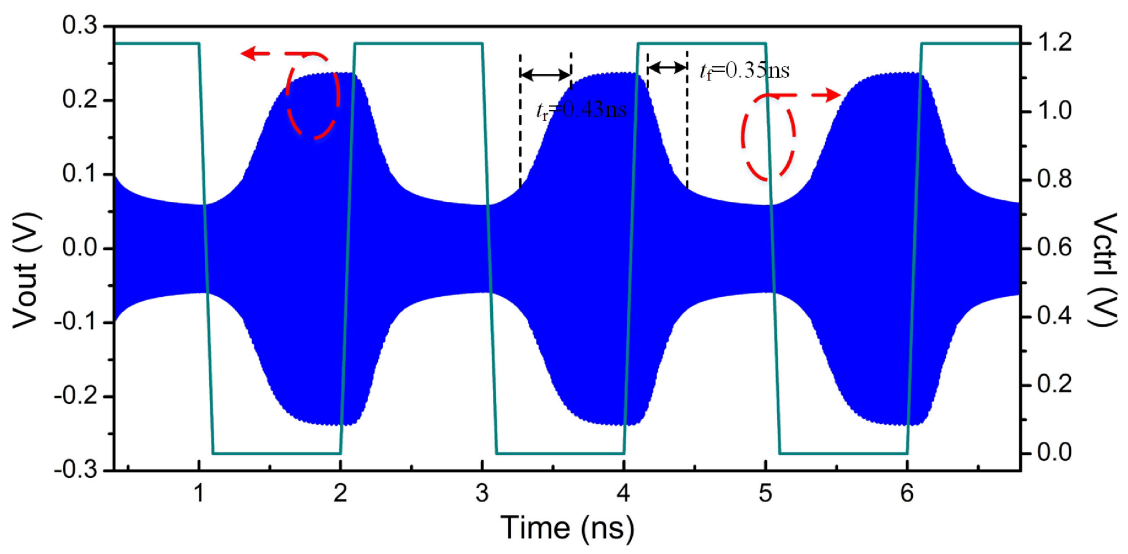

Fig. 11. Simulated switching speed performance. 
The performance of the proposed SPST switch is summarized and compared with recently reported D-band SPST switches in Table II. Compared with the other switches, this SPST switch exhibits competitive performance, especially in terms of insertion loss and core area. Note that the proposed SPST switch is used to realize SPDT T/R switch for TDD-based wideband front-end in the future work rather than realize the on-off keying (OOK) modulation. Therefore, the switching speed is not the key target specification.

Table II. Comparison with recently reported D-band SPST switch

\begin{tabular}{|c|c|c|c|c|c|}
\hline Reference & {$[3]$} & {$[4]$} & {$[5]$} & {$[6]$} & This Work \\
\hline Process & $150 \mathrm{~nm}$ GaAs & $130 \mathrm{~nm}$ BiCMOS & $65 \mathrm{~nm}$ CMOS & $130 \mathrm{~nm}$ CMOS & $65 \mathrm{~nm}$ CMOS \\
\hline Freq. (GHz) & $110-135$ & 135 & $110-170$ & 94 & 120 \\
\hline IL (dB) & $4.1-5$ & 4.7 & $2.5-3.6^{\dagger}$ & 3.8 & 1.9 \\
\hline RL (dB) & $>10$ & 16.3 & $>8.7$ & 11 & $>10$ \\
\hline ISO (dB) & $>15$ & 12.5 & $>19.5$ & 20.1 & $>15.5$ \\
\hline$V_{\text {ctrl }}(\mathrm{V})$ & $0 /-2$ & $0 / 2$ & $0 / 1.2$ & $0 / 2$ & $0 / 1.2$ \\
\hline$P_{1 \mathrm{~dB}}(\mathrm{dBm})$ & N.A. & $6^{\star}$ & N.A. & N.A. & $12.8^{\star}$ \\
\hline $\begin{array}{c}\text { Switching } \\
\text { Speed }(\mathrm{Gb} / \mathrm{s})\end{array}$ & N.A. & 10 & 1 & $20^{\star}$ & $0.5^{\star}$ \\
\hline $\begin{array}{c}\text { Core Area } \\
\left(\mathrm{mm}^{2}\right)\end{array}$ & 0.6888 & 0.128 & 0.013 & 0.0676 & 0.0036 \\
\hline
\end{tabular}

$\dagger$ estimated value from figures; ${ }^{\star}$ simulated

\section{Conclusion}

The proposed switch design using a parallel-stripline swap hybrid coupler with defected ground structure (DGS) was found to be more efficient in comparison to conventional TL, especially at the higher frequency. While the conventional TL fails to deliver low insertion loss at the high frequency, the equivalent capacitance introduced by the coupler gap leads to series resonance condition which results in lower insertion loss at the higher frequency. In addition, the use of DGS results in enhanced equivalent inductance to compensate the parasitic capacitance of the control transistor. Overall, the hybrid coupler provides more degree of design freedom since the capacitance and inductance can be tuned by the structural dimension of the coupler.

\section{Acknowledgments}

This work was supported by the National Natural Science Fundamental of China (61774113, 61574102 and 61404094), the Fundamental Research Funds for the Central Universities, Wuhan University (2042014kf0238 and 2042017gf0052), and the China Postdoctoral Science Foundation (2012T50688). 